\title{
An easy, reproducible and cost-effective method for andrologists to improve the laboratory diagnosis of non- obstructive azoospermia: a novel microcentrifugation technique
}

Rosa Alice Casemiro Monteiro ${ }^{1}$, Juliana Risso Pariz, ${ }^{1,2,3}$, Patrícia de Campos Pieri ${ }^{1}$, Jorge Hallak ${ }^{1,2,3}$

${ }^{1}$ Androscience - Pesquisa Clínica de alta Complexidade e Laboratório de Andrologia, São Paulo, Brasil;

2 Seção de Andrologia - Divisão de Urologia, Faculdade de Medicina da Universidade de São Paulo, São Paulo, Brasil; ${ }^{3}$ Unidade de Reprodução Toxicologia - Departamento de Patologia, Faculdade de Medicina da Universidade de São Paulo, São Paulo, Brasil

\section{ABSTRACT}

This study describes a new method of microcentrifugation as an improved, viable, cost-effective option to the classical Cytospin apparatus to confirm azoospermia. Azoospermic semen samples were evaluated for cryptozoospermia by a centrifugation method similar to that of World Health Organization guidelines (2010; entire specimen centrifuged at $3000 \mathrm{~g}$ for $15 \mathrm{~min}$, and aliquots of the pellet examined). Then, if no sperm were detected, the pellet from that procedure was resuspended in culture medium, centrifuged (2000g for $15 \mathrm{~min}$ ), and the entire pellet spread on a $4 \mathrm{X} 6 \mathrm{~mm}$ area of a slide and stained using the Christmas tree method (Nuclear-Fast solution and picric acid). The entire stained area was examined for the presence or absence of sperm. A total of 148 azoospermic samples (after standard WHO diagnosis) were included in the study and 21 samples (14.2\%) were identified as sperm-positive. In all microcentrifugation slides, intact spermatozoa could be easily visualized against a clear background, with no cellular debris. This novel microcentrifugation technique is clearly a simple and effective method, with lower cost, increasing both sensitivity and specificity in confirming the absence or presence of spermatozoa in the ejaculate. It may represent a step forward of prognostic value to be introduced by andrology laboratories in the routine evaluation of patients with azoospermia in the initial semen analysis.

\section{ARTICLE INFO}

Key words:

Non-obstructive; Azoospermia; Spermatozoa; Semen; Infertility; Male

Int Braz J Urol. 2016; 42: 132-8

Submitted for publication:

February 18, 2015

Accepted after revision:

November 06, 2015

\section{INTRODUCTION}

Non-obstructive azoospermia (NOA) affects approximately $10 \%$ of all men presenting with infertility and is responsible for $80 \%$ in the subgroup of azoospermic men, whereas obstructive azoospermia contributes to $20 \%$ of this subpopulation of infertile men (1). The management of patients with NOA relies on the correct diagnosis, induction of spermatogenesis to try to produce an ejaculate of viable spermatozoa, and finally techniques for sperm procurement, such as micro-dissection testicular sperm extraction, simple sperm extraction (TESE) or Fine Needle Aspiration (FNA), preferentially followed by either sperm or testicular tissue cryopreservation to subsequent 
intracytoplasmic sperm injection (ICSI) use (2-5).

"Should only a few or no spermatozoa be seen at initial evaluation, the sample must be centrifuged and the sediment examined for spermatozoa. The term azoospermia can only be used if no spermatozoa have been found in the sediment" wrote Eliasson 1981 (3). Although apparently simple, the diagnosis of azoospermia has a wide variety of confounding factors including those related to methodology and different evaluation protocols, large errors associated with counting few spermatozoa, the number of microscopy fields to be analyzed, difficulties in examining debris-laden pellets (6). The World Health Organization's recommended changes include, examining fixed uncentrifuged samples and indicating the sensitivity of the counting methods employed; however, existing centrifugation methods necessary for accumulating sufficient number of cells are also included (6).

Whereas that presence of any spermatozoa in a complementary test of azoospermic patient may determine the clinical approach to be adopted, the centrifugation method of cell suspension on slides (Shandon CytoSpin III Cytocentrifuge, Thermo Scientific, Waltham, MA, USA) is widely used to concentrate the ejaculate in a single droplet to enable checking for the presence of spermatozoa in an optical microscope $(7,8)$. The Cytospin apparatus is a bench-top centrifuge with a specially-designed rotor, and sample chambers in which a special micro-slide is vertically placed after being filled with $0.1 \mathrm{~mL}$ of well-mixed whole semen and an equal volume of sterile saline added in situ. This method of diluting the specimen is preferable to using a premixed dilution and minimizes cell-sampling errors, and should be used on specimens in which "no spermatozoa" were detected in a wet preparation. After centrifugation the cells are deposited in a uniform monolayer in a compact area $\left(32 \mathrm{~mm}^{2}\right)$ and even with the use of stains such as nuclear fast red and picroindigocarmine (described as NF-PICS or Christmas Tree stain; Sigma-Aldrich, St. Louis MO, USA) $(9,10)$, is not always easy to identify isolated sperm heads owing to the large amount of cellular debris. Therefore, our group has developed a simple and cost-effective technique as an alternative to the Cytospin method: the microcentrifugation technique to confirm azoospermia (Labnet, Woodbridge NJ, USA). The aim of this study is to demonstrate an alternative method to confirm and improve the laboratory diagnosis of non-obstructive azoospermia.

\section{PATIENTS AND METHODS}

\section{Study population}

A retrospective study was performed involving 148 slides from semen of non-obstructive azoospermic patients in the reproductive age (mean 40.66; standard deviation 9.40) presenting to male infertility evaluation at Androscience, High Complexity Clinical and Research Andrology Laboratories, São Paulo, Brazil, between November 2008 and July 2013. Institutional Review Board approval was obtained from the University of São Paulo Research Ethics Committee and before that all samples were collected after informed consent signature.

\section{Seminal Analysis}

Semen sample was obtained by masturbation after 48 to 72 hours of sexual abstinence. All semen analysis was performed manually by the same investigator (RACM).

After liquefaction, macroscopic and microscopic parameters were analyzed according to World Health Organization (WHO) guidelines of 1999 and $2010(6,11)$. Semen was evaluated for cryptozoospermia by a centrifugation method similar to that in WHO (2010; entire specimen centrifuged at $3000 \mathrm{~g}$ for $15 \mathrm{~min}$, and aliquots of the pellet examined), following by triplicate sediment evaluation $(100 \mu \mathrm{L})$ in all field of Neubauer chamber (6). If any spermatozoa were present in the pellet obtained after this first centrifugation the sample was classified as cryptozoospermic and excluded. In the absence of spermatozoa after the first centrifugation, a standard WHO diagnosis of azoospermia was given and the samples were further alternately processed by the microcentrifugation technique, proposed by this study.

The samples had all of the sediment left after the first centrifugation resuspended in a 
small amount $(100 \mu \mathrm{L})$ of Human Tubal Fluid (Modified HTF Medium, Irvine Scientific, Santa Ana, CA, USA) and centrifuged in a mini-centrifuge (Labnet, Woodbridge NJ, USA) at $2000 \mathrm{~g}$ for 15 minutes. After removal of the supernatant, the pellet was deposited on and gently spread over a glass slide with a pipette in order to cover an area of 2 to $4 \mathrm{~cm}^{2}$.

The slides obtained were then fixed in absolute ethanol for 15 minutes, and air dried. Nuclear-Fast Red solution [73.1mM Aluminum Sulfate (cat\#202614, Sigma Aldrich, St. Louis, MO, USA) and $1.49 \mathrm{mM}$ Fast Nuclear Red (cat\#N8002, Sigma Aldrich, St. Louis, MO, USA) in $50 \mathrm{~mL}$ distilled water], was placed over the slide for 15 minutes, after which the slides were carefully rinsed with distilled water, covered with picroindigocarmine stain $[7.15 \mathrm{mM}$ Indigo Carmine (cat\#18130, Sigma Aldrich, St. Louis, MO, USA) and 50mL of Picric Acid (cat\#92540, Sigma Aldrich, St. Louis, MO, USA)] for 15 seconds, immediately rinsed with absolute ethanol, left to air dry, covered with coverslips and mounted with Entellan (Merck Millipore, Darmstadt, Germany) (8).

The slides were examined at $1000 \times$ magnification in optical microscope (Nikon Eclipse E200, Japan) for the following parameters: sperm presence or absence, sperm integrity, and for the presence of cellular debris.

\section{Statistical analysis}

Statistical analysis was performed using IBM SPSS Statistics 19 for Windows. We calculated the mean of age (years) and frequency of events in the study (\%).

\section{RESULTS}

A total of 148 azoospermic samples (after standard WHO diagnosis) were included in the study. Twenty one samples (14.2\%) were identified as sperm-positive. In addition, intact spermatozoa could be visualized against a clear background, with minimal cellular debris in all slides (Figure-1).

\section{DISCUSSION}

Although intracytoplasmic sperm injection (ICSI) is a remarkably effective technique in allowing fatherhood in men previously considered sterile, sperm retrieval from the epididymis or testis should be the last option to be given to men. Gnoth et al. showed that the source of spermatozoa has not relevant impact on the results of ICSI cycles as long as fresh motile, morphologically normal spermatozoa are used, proposing, in case of cryptozoospermia, to preferentially use ejaculated spermatozoa to prevent those men from an unnecessary testicular biopsy avoiding risks and costs implied (12). In addition, the

Figure 1 - Spermatozoa found after microcentrifugation technique $(2000 \mathrm{~g}$ for 15 minutes), stained by nuclear fast red and picroindigocarmine (described as NF-PICS or Christmas Tree stain) in an azoospermic man. We observed clear slides obtained with sperm integrity preserved, without cellular debris.

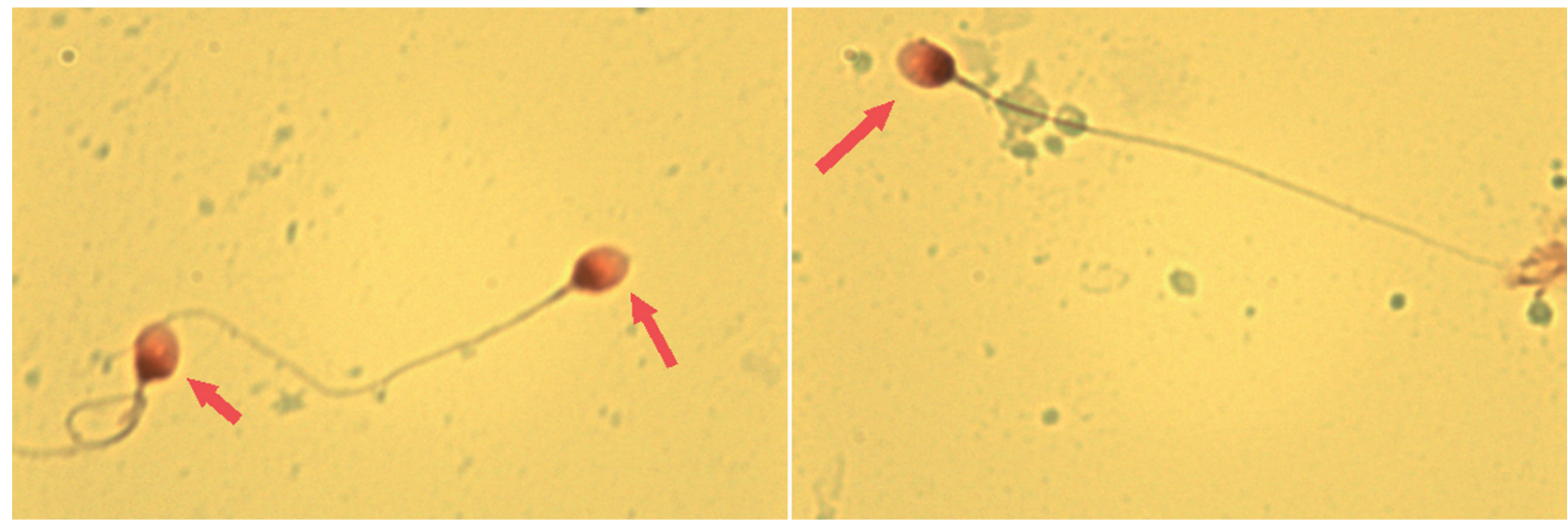

1000x in immersion oil. 
use of a single sperm found in ejaculate in ICSI resulting in successful pregnancy was demonstrated by Desai et al. (13). Thus, azoospermia requires a diligent search for reversible factors and treatment to restore natural fertility and a pragmatic 'default to ICSI' can be avoided (14).

Semen analysis is the single most important diagnostic tool method for the assessment of male infertility and the gold standard according to the World Health Organization. The WHO guidelines offer detailed advice on every aspect of semen analysis and consequently has become the gold standard for the field (6). According to these guidelines, a patient is diagnosed as azoospermic when few or no sperm cells are found in wet preparations, the sample is centrifuged and there is also absence of sperm in the sediment (3). However, the presence or not of spermatozoa in the sediment depends on the centrifugation time and speed and on how much of the sediment is examined and how thoroughly (6). No technique more stringent is proposed by the WHO guidelines to characterize the sample further after the routine seminal analysis does not identify any spermatozoa.

Shandon Cytospin emerged in the 1970s as an apparatus suited for cell concentration and has since been used for sediment analysis of many fluids. In the late 1980s it was first used to analyze the presence of germ cells exfoliate in semen samples and identification of carcinoma in situ of the testis (15). Afterwards Cystospin was introduced in Andrology laboratories as an additional test to confirm azoospermia. Despite being a simple and rapid method, processing the sample often causes damage to the structure of the sperm cells, such as the separation of the heads from mid piece and tail. Furthermore, the presence of cellular debris makes a detailed analysis difficult and time-consuming. In the view of these disadvantages, the microcentrifugation technique developed by our group proved to be as effective and possibly increased the sensitivity and specificity in confirming azoospermia, beyond the cost 5 times lower, since it uses the whole sediment obtained after the first centrifugation, minimizing cell losses but maximizing debris washout.

We purposed to demonstrate a method detection of single sperm cells in semen sediments as Cytospin, but with visualization and identification of isolated sperm cells much easier. From the technical standpoint, the differences between the two methods presented here are: a second wash to remove cellular debris, the rotational speed applied to the sample, and the way the final sample is "deposited" on the slide. More important than the time and rotation speed, the new technique proposed uses another wash with culture medium which can contribute to both the concentration of the sediment and the removal of cellular debris. The centrifugation and pellet resuspension technique is widely used in laboratory routine to eliminate cell debris and was applied in our method. Additionally, the sample deposition on slide is carried out differently: while Cytospin method concentrated all sample in a small area of slide, the microcentrifugation allows the sample to spread over a larger area of slide surface with a pipette.

There is no consensus on which rotation is best for the processing of azoospermic semen samples. Whereas the Cytospin centrifuge uses $700 \mathrm{~g}$ for five minutes, the microcentrifugation proposed by us used $2000 \mathrm{~g}$ for 15 minutes. In the literature, the speed centrifugation of semen samples remains controversial. Corea and colleagues (16) showed that centrifugation should apply at least $1000 \mathrm{~g}$ for 5 minutes in order to find sperm cells in suspected cases of azoospermia but the WHO guidelines (6) suggests centrifugation at $3000 \mathrm{~g}$ for 15 minutes for samples in which no sperm cell is found. Other studies showed that centrifugation at $1000 \mathrm{~g}$ for 15 minutes is effective $(17,18)$. Our hypothesis of $2000 \mathrm{~g}$ for 15 minutes was based on laboratory practice and literature, considering that (I) little centrifugation time was not enough for the pellet formation and (II) $3000 \mathrm{~g}$ damaged the sperm integrity.

The NF-PICS or Christmas Tree stain is one of the most widely used for histological tests for the identification of sperm in sexual assault cases (10). With this stain it is possible to color both the post-acrosomal region of the sperm head, that stains pink, and the acrosome itself, that stains light pink, while cell debris are colored green, allowing easy differentiation of spermatozoa from cellular debris and thus facilitating the reading of the slide. Other cellular dyes, such as eosin-nigrosin, Hoechst 33342 fluorescent stain and Diff-Quick can be suggested to replace the NF-PICS $(6,19)$. Therefore, as azoospermia is a complex diagnosis and subjected to doubts, it is important to preserve the slide with mounted coverslip 
after staining not only for subsequent reading but also to make it available for a second opinion review by other professionals.

In conclusion, the microcentrifugation method developed by our group showed to be a simple, effective, and low cost technique able to increase the sensitivity of confirming azoospermia, an important step of prognostic value to be used hand in hand with clinical and surgical approaches aimed to reverse azoospermic state.

\section{ABBREVIATIONS}

NOA = Non-obstructive azoospermia

TESE $=$ Testicular sperm extraction

FNA $=$ Fine Needle Aspiration

ICSI = Intracytoplasmic sperm injection

WHO $=$ World Health Organization

NF-PICS $=$ Nuclear fast red and picroindigocarmine

\section{ACKNOWLEDGEMENTS}

We thank the Androscience for financial support.

\section{CONFLICT OF INTEREST}

None declared

\section{REFERENCES}

1. Jarow JP, Espeland MA, Lipshultz LI. Evaluation of the azoospermic patient. J Urol. 1989;42:62-5.

2. Abdel Raheem A, Garaffa G, Rushwan N, De Luca F, Zacharakis E, Abdel Raheem T, et al. Testicular histopathology as a predictor of a positive sperm retrieval in men with non-obstructive azoospermia. BJU Int. 2013; 111:492-9.

3. Eliasson R: The testis. New York, NY: Raven Press; 1981.

4. Schlegel PN, Palermo GD, Goldstein M, Menendez S, Zaninovic N, Veeck LL, et al. Testicular sperm extraction with intracytoplasmic sperm injection for nonobstructive azoospermia. Urology. 1997; 49:435-40.

5. Su LM, Palermo GD, Goldstein M, Veeck LL, Rosenwaks Z, Schlegel PN. Testicular sperm extraction with intracytoplasmic sperm injection for nonobstructive azoospermia: testicular histology can predict success of sperm retrieval. J Urol. 1999; 161:112-6.

6. WHO. World Health Organization: Laboratory manual for the examination and processing of human semen. 5th ed. Geneva: WHO Press; 2010.
7. Coburn M, Wheeler T, Lipshultz LI. Testicular biopsy. Its use and limitations. Urol Clin North Am. 1987; 14:551-61.

8. Hendin BN, Patel B, Levin HS, Thomas AJ Jr, Agarwal A. Identification of spermatozoa and round spermatids in the ejaculates of men with spermatogenic failure. Urology. 1998; 51:816-9.

9. Allery JP, Telmon N, Mieusset R, Blanc A, Rougé D. Cytological detection of spermatozoa: comparison of three staining methods. J Forensic Sci. 2001; 46:349-51.

10. Simons JL, Vintiner SK. Effects of histological staining on the analysis of human DNA from archived slides. J Forensic Sci. 2011; 56:S223-8.

11. WHO. World Health Organization: WHO Laboratory manual for the examination of human semen and sperm-cervical mucus interaction. 3rd ed. Cambridge: Cambridge University Press; 1999.

12. Gnoth C, Markhinin V, Maxrath B, Skonieczny T, Friol K, Roos J, et al. Impact of sperm cell source on the results of intracytoplasmic sperm injection. Arch Gynecol Obstet. 2015; 291:663-9.

13. Desai N, Goldberg J, Austin C, Sabanegh E, Falcone T. Cryopreservation of individually selected sperm: methodology and case report of a clinical pregnancy. J Assist Reprod Genet. 2012; 29:375-9.

14. McLachlan RI. When is azoospermic infertility treatable without intracytoplasmic sperm injection? Clin Endocrinol (Oxf). 2013 78:176-80

15. Giwercman A, Marks A, Skakkebaek NE. Carcinoma-in-situ germ-cells exfoliated from seminiferous epithelium into seminal fluid. Lancet. 1988; 1:530.

16. Corea M, Campagnone J, Sigman M. The diagnosis of azoospermia depends on the force of centrifugation. Fertil Steril. 2005; 83:920-2.

17. Mortimer D: Practical Laboratory Andrology: Oxford University Press; 1994.

18. Manual on basic semen analysis, 2002: ESHRE, SIGA. Available at. http://www.fivfrance.com/pro/pdf_NAFA_ESHRE2002.pdf.

19. Beyer CE, Kayler B, Osborne E, McLachlan R, Osianlis T. Supersensitive fluorescent semen analysis: validation on azoospermic and oligozoospermic samples. Fertil Steril. 2012; 98:843-8.e1.

Correspondence address: Juliana Risso Pariz, MD Androscience - Pesquisa Clínica de alta Complexidade e Laboratório de Andrologia, São Paulo, Brasil R. Joaquim Floriano, 533 cj 904 São Paulo, SP, 04534-011, Brasil Telephone: +55 11 3073-0623 E-mail: lab@androscience.com.br 
In this issue of Int Braz $\mathrm{J}$ Urol, a paper by Monteiro and colleagues reports on a novel semen analysis method to increase precision in the diagnosis of azoospermia (1). This paper is important given the recent changes in the World Health Organization (WHO) laboratory methods for evaluation of human semen (2) and the current discussion on how to count sperm properly (3). This editorial commentary is aimed to highlight Monteiro and colleagues findings and to expand the discussion on the clinical importance of laboratory andrology.

Azoospermia is a laboratory diagnosis of ejaculates that lack spermatozoa (4). Despite being a descriptive term that does not imply any specific underlying cause, the absence of spermatozoa in a semen analysis has many ramifications for both clinical practice and research. The reliable diagnosis of azoospermia is important not only for male infertility and to ensure the success of vasectomy but also for assessing efficacy of male contraceptive trials. Moreover, the confirmation for the presence of semen in forensic studies is based on the direct identification of spermatozoa.

Seminal fluid is a complex mixture of secretions from the seminal vesicle, the prostate gland and the combined contribution of the epididymis, testicles and bulbourethral glands (5). An average male ejaculates around $3.2 \mathrm{~mL}$ of semen. Each milliliter contains approximately 64 million spermatozoa (6). This number can vary with the age of the male, and may be affected by other factors, including medical conditions, genetic background, ejaculatory abstinence and life-style habits such as diet, tobacco and illicit drug use (7). On the contrary, azoospermia affects approximately $1 \%$ of males in the general population and 10-15\% of those facing infertility (8).

In the context of infertility, men with azoospermia do not have an unattainable potential to initiate a pregnancy. From the laboratory standpoint, the assessment of an initially azoospermic ejaculate should be followed by the examination of the pelleted semen to exclude cryp- tozoospermia, which is defined by the presence of very small number of live sperm (8). This is important because the finding of live sperm may allow intracytoplasmic sperm injection to take place without the need of surgical sperm retrieval. Since the diagnosis of azoospermia or cryptozoospermia is based upon semen analysis, proper laboratory techniques are crucial to reduce analytical errors and enhance precision (9).

Accuracy, the degree to which the measurement reflects the true value, and precision, the reproducibility of the results, are important for clinicians who rely upon the values provided by the laboratory to direct the further work-up and to counsel men seeking fertility (9). Interestingly, data from surveys of laboratory practice in the United States and the United Kingdom revealed that semen analysis techniques are poorly standardized. Interestingly, less than half of the laboratories providing semen analysis services have implemented quality control practices and only about 5\% fully complied with the WHO manual for the laboratory assessment of human semen $(10,11)$.

Owed to its complex nature, we have advocated that semen analysis should be ideally carried out in Andrology Laboratories equipped with experienced technicians and validated systems, and enrolled in internal and external quality control programs $(3,8,9,12)$. In this current issue of Int Braz J Urol, Monteiro and colleagues shed light on this very same issue (1). The authors studied semen specimens from men with nonobstructive azoospermia, all of which were negative for the presence of sperm after processing according to the new WHO guidelines. Of note, the WHO method recommends centrifugation of a $1 \mathrm{~mL}$ aliquot of semen for 15 minutes using high $g$ force and examination of two aliquots of the pelleted semen (2). The use of high centrifugation force is warranted because the accuracy of any centrifugation protocol using low $\mathrm{g}$ forces in pelleting all spermatozoa in a given ejaculate is uncertain (13). However, the aforementioned WHO method is flawed by not 
recommending examination of the whole specimen. Furthermore, the pelleted semen gives rise to large amount of debris that might make it difficult to identify isolated spermatozoa (13). Monteiro et al. proposed an additional centrifugation step using high g force, followed by examination of the whole pellet deposited in a large area of a microscope glass slide (1). For this, the slides were fixed and stained with nuclear fast red and picroindigocarmine. Interestingly, clear slides were obtained with minimum debris, and isolated spermatozoa were identified in about 14\% of the specimens evaluated. These patients were reclassified as having cryptozoospermia, with obvious diagnostic and management implications.

Although a comparative analysis with other centrifugation and staining techniques has not been included, Monteiro and colleagues' method was clearly superior to the WHO method for evaluating azoospermic specimens. The finding of any sperm in a semen analysis meticulously performed, such as the one object of this commentary, has important implications for men with nonobstructive azoospermia. It is not only a proof that residual sperm production exists, thus allowing the affected men to achieve biological offspring with the aid of assisted reproductive technology, but also imply that sperm retrieval is likely to be successful whenever required.

Collectively, the study of Monteiro and colleagues highlights the importance of semen analysis in the context of azoospermia and the role of Andrology Laboratories to improve precision and reproducibility of reported results.

Sandro C. Esteves, $M D, P h D$ Medical and Scientific Director, ANDROFERT Center for Male Reproduction Av. Dr. Heitor Penteado 1464 Campinas, SP, 13075-460, Brazil E-mail: s.esteves@androfert.com.br 